\title{
Echocardiography accurately predicts pulmonary hypertension in patients with advanced lung disease
}

\author{
Silvia Cottini ${ }^{1}$, Christian Benden ${ }^{2}$, Lars C. Huber ${ }^{2,3}$ and Mattia Arrigo ${ }^{4^{*}}$ (D)
}

Keywords: Pulmonary hypertension, Echocardiography, Advanced lung disease, Transplantation, Right heart catheterization

Pulmonary hypertension (PH) may be observed in many different conditions, including advanced heart or lung disease [1]. $\mathrm{PH}$ is defined as elevation of the mean pulmonary arterial pressure $(\mathrm{mPAP}) \geq 25 \mathrm{mmHg}$, measured by right heart catheterization (RHC). Since procedure-related risks and costs are not negligible, RHC is not routinely performed in patients with advanced lung disease during initial assessment and follow-up. Echocardiography is commonly used as screening tool for the presence of $\mathrm{PH}$, but might be of limited diagnostic value, in particular in patients with lung disease [2]. Empirical formulas were proposed to calculate the mPAP starting from systolic pulmonary arterial pressure (sPAP) as assessed by echocardiography $[3,4]$. It has been suggested that both the Chemla formula $(\mathrm{mPAP}=\mathrm{sPAP} \times 0.61+$ $2 \mathrm{mmHg}$ ) and the Syyed formula (mPAP $=\operatorname{sPAP} \times$ $0.65+0.55 \mathrm{mmHg}$ ) might accurately estimate the mPAP [5]. The aim of our study was to assess the accuracy of these echocardiography-based formulas to estimate the invasively measured MPAP in a cohort of patients with advanced lung disease. Estimation of SPAP by echocardiography was performed according to current recommendation by adding to the right ventricular/right atrial pressure gradient (based on Doppler-measured tricuspid regurgitation velocity) the estimated right atrial pressure (based on inferior vena cava diameter and collapsibility). A total of 96 consecutive patients undergoing lung transplant evaluation between $03 / 2000$ and 10/2015 were included. Data from RHC and echocardiography performed the same day were available for the whole cohort.

Spearman's rank-order correlation was run to assess the relationship between RHC-measured mPAP and calculated mPAP. There was a strong positive correlation between both RHC-mPAP and ChemlamPAP (rs $=0.909, p<0.001$; Fig. 1a) and between RHC-mPAP and Syyed-mPAP ( $\mathrm{rs}=0.904, p<0.001$; Fig. 1b).

Inspection of both diagrams suggests that the correlation between echocardiographic data and invasive pulmonary hemodynamics is of particular strength in patients with moderate elevation of pulmonary pressure. Whether echocardiography-based formulas are of similar accuracy to estimate pulmonary pressure of $\mathrm{PH}$ in patients with severe $\mathrm{PH}$ remains unclear. However, this might be of minor relevance since, as shown in our cohort, only a minority of patients with advanced lung disease present with a mPAP $>50 \mathrm{mmHg}$. In these patients, further invasive assessment of pulmonary hemodynamics may be indicated. For all other patients our data emphasize that echocardiography is an accurate tool to estimate pulmonary pressure in patients with advanced lung disease.

\footnotetext{
* Correspondence: mattia.arrigo@usz.ch

${ }^{4}$ Department of Cardiology, University Hospital Zurich, Raemistrasse 100, 8091 Zurich, Switzerland

Full list of author information is available at the end of the article
} 

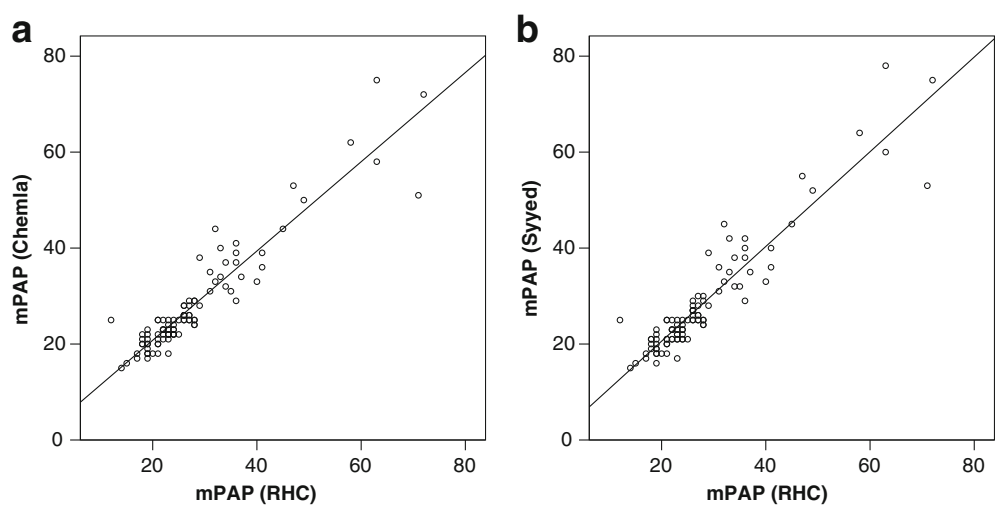

Fig. 1 Correlation between RHC-measured mPAP and echocardiographic-estimated mPAP using the Chemla formula (a) and the Syyed formula (b). Values expressed as $\mathrm{mmHg}$

\section{Abbreviations}

mPAP: Mean pulmonary arterial pressure; PH: Pulmonary hypertension; RHC: Right heart catheterization; SPAP: Systolic pulmonary arterial pressure

\section{Acknowledgements}

Not applicable.

\section{Funding}

Departmental funds.

\section{Availability of supporting data}

All supporting data are available upon request.

\section{Authors' contributions}

SC participated in the design of the study, collected the data, and drafted the manuscript. CB participated in the design of the study and critically revised the manuscript. LCH participated in the design of the study and drafted and revised the manuscript. MA performed statistical analyses and drafted and revised the manuscript. All authors gave final approval of the manuscript. All authors participated in data analysis and manuscript draft and revision. All authors read and approved the final manuscript.

\section{Competing interests}

The authors declare that they have no competing interests.

\section{Consent for publication}

Publication was authorized by the local Ethical Committee (BASEC 201601642).

\section{Ethics approval and consent to participate}

The study was performed according to the principles of the Declaration of Helsinki and was authorized by the local Ethical Committee (Kantonale Ethikkommission Zürich, Switzerland: BASEC 2016-01642).

\section{Publisher's Note}

Springer Nature remains neutral with regard to jurisdictional claims in published maps and institutional affiliations.

\section{Author details}

${ }^{1}$ Intensive Care Unit, University Hospital Zurich, Zurich, Switzerland. ${ }^{2}$ Division of Pulmonology, University Hospital Zurich, Zurich, Switzerland. ${ }^{3}$ Clinic for Internal Medicine, City Hospital Triemli, Zurich, Switzerland. ${ }^{4}$ Department of Cardiology, University Hospital Zurich, Raemistrasse 100, 8091 Zurich, Switzerland
Published online: 25 May 2017

\section{References}

1. Arrigo $M$, Huber LC. Passive pulmonary hypertension: more than hydrostatics. Chest. 2014;145:413.

2. Janda S, Shahidi N, Gin K, Swiston J. Diagnostic accuracy of echocardiography for pulmonary hypertension: a systematic review and meta-analysis. Heart. 2011;97:612-22. http://heart.bmj.com/cgi/doi/10.1136/ hrt.2010.212084

3. Chemla D, Castelain V, Provencher S, Humbert M, Simonneau G, Hervé P. Evaluation of various empirical formulas for estimating mean pulmonary artery pressure by using systolic pulmonary artery pressure in adults. Chest. 2009;135:760-8.

4. Syyed R, Reeves JT, Welsh D, Raeside D, Johnson MK, Peacock AJ. The relationship between the components of pulmonary artery pressure remains constant under all conditions in both health and disease. Chest. 2008;133:633-9.

5. Aduen JF, Castello R, Lozano MM, Hepler GN, Keller CA, Alvarez F, et al. An alternative echocardiographic method to estimate mean pulmonary artery pressure: diagnostic and clinical implications. J Am Soc Echocardiogr. 2009; 22:814-9. 